\title{
Urachal Remnant
}

National Cancer Institute

\section{Source}

National Cancer Institute. Urachal Remnant. NCI Thesaurus. Code C123255.

Remaining collapsed and obliterated urachal tissue between the bladder dome and umbilicus. 